\title{
Identification of viruses in Acute Lower Respiratory Infections (ALRI) in Lao People's Democratic Republic
}

\author{
Anne-Charlotte Sentilhes ${ }^{1,2^{*}}$, Vimatha Xaysitthideth ${ }^{1}$, Sareth Rith ${ }^{3}$, Somvay Ongkhamme ${ }^{1}$, Thongchanh Sisouk , \\ Darouny Phonekeo ${ }^{1}$, Jean-Jacques Bernatas ${ }^{2}$, Vincent Deubel ${ }^{3}$, Philippe Buchy ${ }^{3}$, Paul Brey ${ }^{2}$, \\ Phengta Vongphrachanh ${ }^{1}$
}

From Institut Pasteur International Network Annual Scientific Meeting

Hong Kong. 22-23 November 2010

\section{Background}

Acute respiratory infections are a major cause of mortality and morbidity worldwide. Information on viral etiology in ALRI from Lao PDR is limited. The aim of the present study was to use Multiplex PCR/RT-PCR methods for the detection of the major respiratory viruses.

\section{Methods}

Nasal/throat swab specimens were collected from patients enrolled in the ALRI surveillance programme within 2 hospitals, one in Vientiane Capital (Setthathirat Hospital) and the second one in Luang Prabang. From each sample, viral RNA was extracted and amplified by using 5 multiplex PCR/RT-PCR targeting 18 respiratory viruses.

\section{Results}

Between 2009 and 2010, Multiplex PCR / RT-PCR detected respiratory viruses in 111 (54.7\%) of 203 samples. Single virus was detected in $44.8 \%(91 / 203)$ and virus co-infections were observed in $9.9 \%(20 / 203)$. Rhinovirus (40.5\%), human Respiratory Syncytial virus (hRSV; 27.9\%), and Influenza A virus (9.0\%) were the most frequently detected viruses. Adenovirus and human Metapneumovirus were detected in $8.1 \%$ and $6.3 \%$ of ALRI specimen, respectively. Influenza $C$ virus and SARS-coronavirus were not detected during the study period. Children $<5$ years represented $50 \%$ of patients identified.

\section{Conclusion}

Our results provide new documentation about etiology of respiratory virus diseases in Lao People's Democratic Republic. In this context, multiplex PCR/RT-PCR offers a sensitive and reasonably priced diagnostic method for common respiratory viruses.

\section{Acknowledgements}

Supported by the French Development Agency through the Surveillance and Investigation of Epidemic Situations in Southeast Asia (SISEA) project.

\section{Author details}

${ }^{1}$ National Center for Laboratory and Epidemiology, Vientiane, Lao PDR. ${ }^{2}$ Institut Pasteur in Laos, Vientiane, Lao PDR. ${ }^{3}$ Institut Pasteur in Cambodia, Phnom Penh, Cambodia.

Published: 10 January 2011

* Correspondence: acsentilhes@gmail.com

${ }^{1}$ National Center for Laboratory and Epidemiology, Vientiane, Lao PDR

Full list of author information is available at the end of the article

doi:10.1186/1753-6561-5-S1-P74

Cite this article as: Sentilhes et al:: Identification of viruses in Acute Lower Respiratory Infections (ALRI) in Lao People's Democratic Republic. BMC Proceedings 2011 5(Suppl 1):P74. 